\title{
Pharmacogenomics/Genetics Biomarker Supplemental Qualifiers Dataset
}

National Cancer Institute

\section{Source}

National Cancer Institute. Pharmacogenomics/Genetics Biomarker Supplemental

Qualifiers Dataset. NCI Thesaurus. Code C147232.

A dataset containing supplemental information, specifically non-standard variables, to parent records in the pharmacogenomics/genetics biomarker domain. 